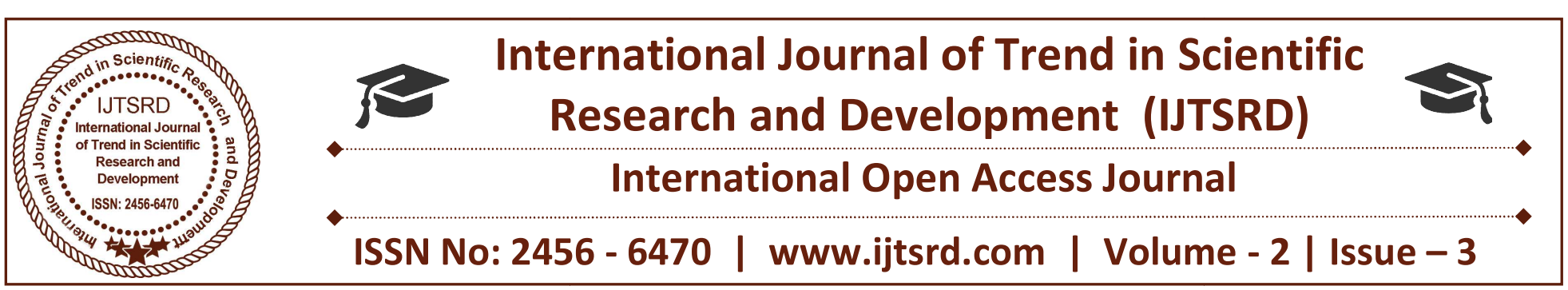

\title{
Raspberry Pibot Manipulated with Skid-Steered Mechanism for Multi-Environment Surveillance
}

\author{
${ }^{1}$ Amrutha Mohanan. K. V, ${ }^{1}$ Hema. E. K, Monisree. S, ${ }^{2}$ Audline Beena. S. P \\ ${ }^{1}$ Student, ${ }^{2}$ Assistant Professor \\ Department of Computer Science and Engineering, \\ Sri Muthukumaran Institute of Technology, Mangadu, Chennai, India
}

\begin{abstract}
In today's world, everyone is worried about their safety due to increase in crime rate. This has led to an increase in the importance of surveillance systems. In this project, raspberry pi serves as the heart of the robot which in turn is used to implement the real-time surveillance system possible within a local network. The major factor enhanced in this project is the skidsteered mechanism manipulated over the motor controlling the robotic movement. This particular mechanism has the major role in imparting an equilibrium movement of the robot in the rough terrain. Depending on the obstacles identified in the area the pibot maintains the area map and also update its frequency as per the obstacles encountered. Further the environmental conditions monitored by the sensor unit will benefit in obtaining a clear idea of the remote area .A single camera is installed on a robot and the robot can move in all directions to take photos in different angles. The $\mathrm{Pi}$ has the capability of installing and processing high resource software's which makes it possible to accomplish the objectives of live streaming \& controlling the robot. The live streaming is proficient by using the mjpeg streamer and the server-client model is made using java.
\end{abstract}

Keywords: Surveillance, Pibot, Raspberry pi, Local network, Skid-steered, mjpeg streamer, server-client model

\section{INTRODUCTION}

Today each and every one is concerned about their security since the growth rate of crime has increased.
This caused people to have started to consider the significance of surveillance systems. Majority of the people are doing IP based installations rather than the analog because of it is accessible from anywhere. In order to make the IP-based systems affordable for the people having a low budget, we need to develop a system which is cost effective and portable. This project uses raspberry pi model ' $\mathrm{B}$ ' for making this real-time surveillance possible. The $\mathrm{Pi}$ has the capability of installing and processing high resource software's which makes it possible to accomplish the objectives of live streaming \& controlling the robot. Basically, the surveillance systems are built up with multiple cameras which are placed in different angles of view to track human objects[1]. Particularly the tracking task is needed on cameras for dynamic objects which increases the number of cameras used in the system. An intelligent surveillance system with multiple cameras is complicated and costly. To overcome this we use a single camera is installed on a robot and the robot can move all directions to take photos in different angles. This system is very flexible to monitor any living object with the help of the PIR sensor and it is more suitable for surveillance systems. The wireless control provides additional benefits including increased flexibility and reduced installation cost.

\section{CURRENT SCENARIO SURVEILLANCE SYSTEMS}

The border guarding forces are patrolling the border seriously, but it is not possible to watch the border at 
each and every moment. An essential requirement of this situation is a robot which automatically detects trespassers in the border and report it to the nearby border security control unit.

Two types of such systems are available that are Analog and IP-based video Surveillance systems[2]. The analog systems are somewhat less expensive and easy to operate than the IP-based systems. But it has some limitations such as to cover a larger area we need to deploy the bigger number of cameras and once the cameras are deployed at a particular place it is very cumbersome as well as complicated to shift them to a different location since the system is wired[3]. According to the study of the Axis Communications, the majority of the people are doing IP based installations rather than the analog. This is because of the reason that IP-based video surveillance provides better picture quality and is also beneficial in terms of scalability and flexibility. But working with the IP-based systems requires some networking knowledge and people having a low budget are unable to take advantage of the IP-based systems since these systems are way too expensive than the analog ones. So it is clear that even if the presently available IPbased system overcomes some of the limitations of the analog systems but the camera requirement, complicated operation and cost is still a drawback of these systems. Different kinds of sophisticated electronic devices including surveillance alarm, Closed Circuit Television (CCTV) surveillance etc., is flooding the market at a greater pace. With the advancement in technology, locations can be monitored from remote places at any time, from the outside world.

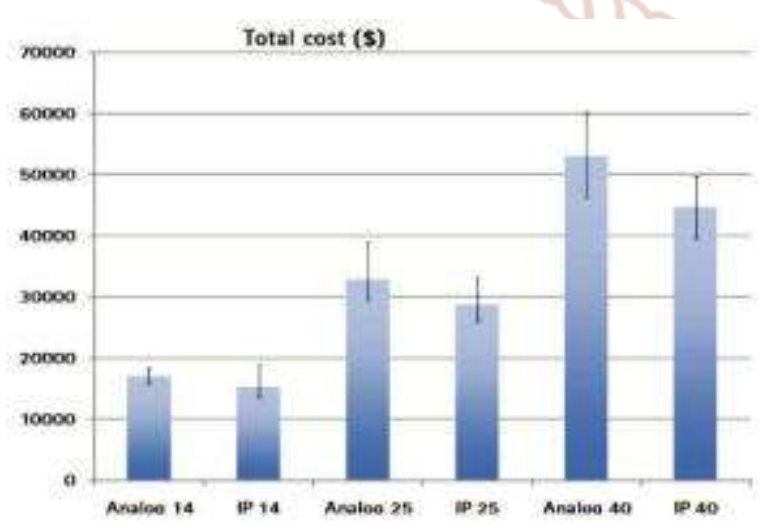

Figure 1. Total cost per camera layout alternative

\section{WHY TO CHOOSE RASPBERRY PI}

The Raspberry Pi is a mini-computer also known as SBC (Single Boarded Computer) [4] and the Arduino is a programmable microcontroller whose functionality depends on the way it is programmed. Comparing Arduino and raspberry pi will be like comparing a mere calculator to a full-fledged laptop [5]. The Arduino is a quite low power microcontroller which provides control over hardware [6]. With the help of the Arduino Integrated Development Environment, programs $(<32 \mathrm{~Kb})$ are interfaced with a hardware like switches, sensors, LCDs, the internet, other microcontrollers, etc. On the other hand, Raspberry $\mathrm{Pi}$ is intended to function smoothly even while using high resource software. Ethernet, video and audio processing, large quantities of RAM and quite a large amount of storage space, makes it a mini-computer [7]. It runs a complete operating system (OS) like Linux and various other flavors of Linux as well as Android. It can also develop programs within those operating systems that can control the functions of the system and the IO that are made available. Raspberry Pi can do everything an Arduino can but in a complex way. Nevertheless, the

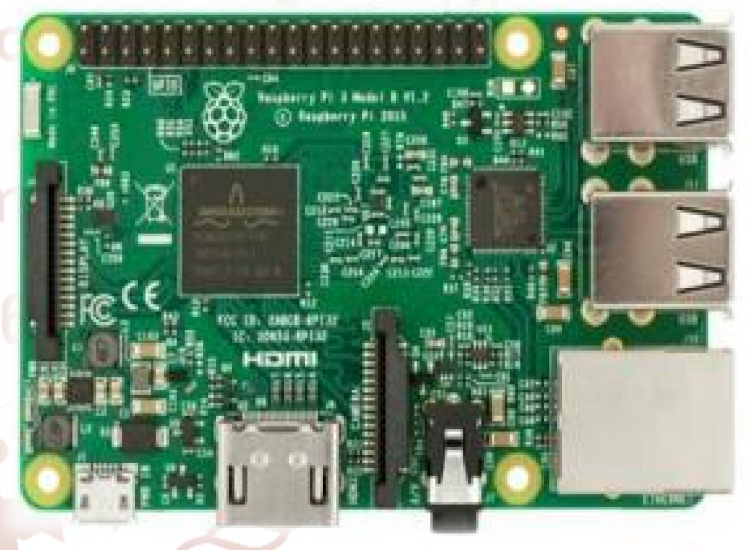

Arduino is not as powerful as the Pi. The Arduino sends data to PC or Pi via serial.

\section{Figure 2: Raspberry pi model B}

Arduino and Raspberry PI might appear alike because they both are tiny little circuit boards with some electronic chips and pins on them to make it function but they are very different devices all together. In fact, Arduino is a low power micro-controller; not a mini-computer like the raspberry pi . A microcontroller is just a minor part of a computer, and only provides a part of the functionality of the Raspberry $\mathrm{Pi}$.The Arduino is programmable with $\mathrm{C}$ language, but it cannot run a complete operating system (OS), whereas raspberry pi can run the 
complete operating system like Linux and Android[7]. The Raspberry $\mathrm{Pi}$ has Python as the main programming language, but can also run $\mathrm{C}, \mathrm{C}++$, Java, Ruby, Perl. The projects made in it are more of software-based rather being hardware based. It can yet do a hardware control-based project with the GPIO pins it has onboard. Arduino is an open-source hardware/ development board designed around an 8bit Atmel AVR microcontroller or a 32-bit Atmel ARM. It has included USB interface, 6 analog input pins, as well as 14 digital I/O pins [7][8]. Proper Arduinos have used the mega AVR series of chips, specifically the ATmega8, ATmega168, ATmega328, ATmega 1280, and ATmega2560. The Arduino has an integrated development environment (IDE) which is a cross-platform application written in Java so that it can be used on any machine.

Raspberry Pi comprise a complete operating system loaded on an SD card, audio out, HDMI and RCA video output and an Ethernet port [7][8]. An Arduino is a picture- perfect for electronics projects. It contains various input and output that can be directly connected to components, sensors and is extremely easy to use.

The Raspberry is considerably more complex for simple electronics projects like just flashing an LED. While on the other hand using the Arduino, it just comprises of connecting an LED and resistor to two pins and simply uploading about 8-10 lines of code. Beside on Raspberry $\mathrm{Pi}$ if the operating system is already installed then there is only a need to install some libraries to control the GPIO pins.

\section{SYSTEM OVERVIEW}

Basically, two gear motors are sufficient to produce the movement of the pi robot and the motor driver module is used to supply enough current to drive two gear motors which protect the Raspberry-pi module from the damage. The major advantage of using the minimum number of gear motor is minimizing the power consumption. Skid-steered vehicles are driven by changing the velocities of the right and left wheels or tracks independently. When vehicles on both sides are rotating at the same speed, the vehicle moves straight ahead. As soon as wheels of one side become slower, the wheel is forced to rotate about an axis, which is outside the wheels of the vehicles. The amount of skid depends on the radius of curvature, i.e., the speed difference between the left and right- side wheels or tracks and increases with decreasing radius of curvature.

Pibot has two infrared sensors which are used to sense the obstacles coming on both sides of robot path. It will move in a particular direction and when the obstacle coming in its path, it will turn to the opposite direction. Besides, the PIR sensor is used to detect the

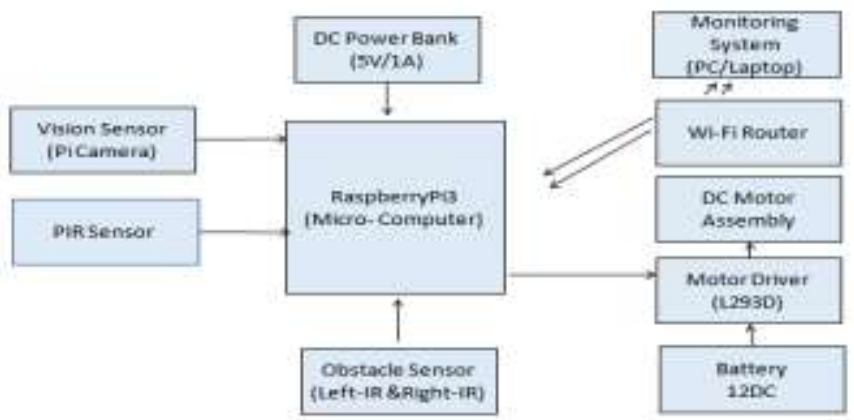

presence of living object in the robotic environment, which in turn to triggers the visual sensor (pi camera) then capture the image or video and store it in the web server.

\section{figure 3. The block diagram of the Pi robot for a surveillance system}

\section{A. ROBOTIC MOVEMENT CONTROL:}

The Pibot 's movement has the major significance in this case. the movement depends on the type of terrain that the robot moves, hence it has become necessary to analyze the environment and make dynamic changes in the movement [9]. The major difficulties arise when the robot is made to move on the rough terrain. The better way to Manage this situation is by regulating the torque of the wheels and assessment the slip probability of each wheel.

As a result of its implementation, the robot moves over a predetermined path, detect and avoid obstacles creates a local map of the area and frequently update the map and determine its own location [10]. Unlike the existing analogs, the focus has not been done on the improving of the mechanical component of the platform and increasing its mobility degree, but on the expansion of sensory systems capabilities [11]. This allows for the robot to decide beforehand the course of the further action to be taken. 


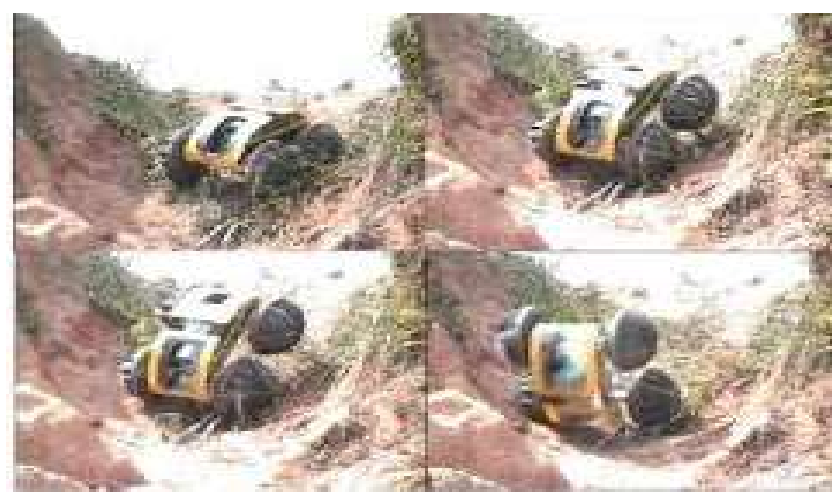

Figure 4: The robot experimented in rough terrain

\section{B. DEVELOPMENT OF AREA MAP:}

When the robot moves over an unknown territory with different terrain, the nearby obstacles might not be in the visibility zone of rangefinder and vision system. In such a situation the robot is intended to rely on the sensor data for obstacle detection, but there is a chance that the obstacle may not fall within the coverage area of the sensing device [12]. Therefore, the best method to overcome this situation will be to develop an area map for the territory.

The robot moves in the open space in different terrain, we use GPS module to define its position in real time. If the device cannot give the signal or when the signal losses, the coordinates of the robot is calculated considerably [12] [13]. Based on this the map for a particular area can be updated henceforth the robot finds it easy to move in equilibrium with the movement.

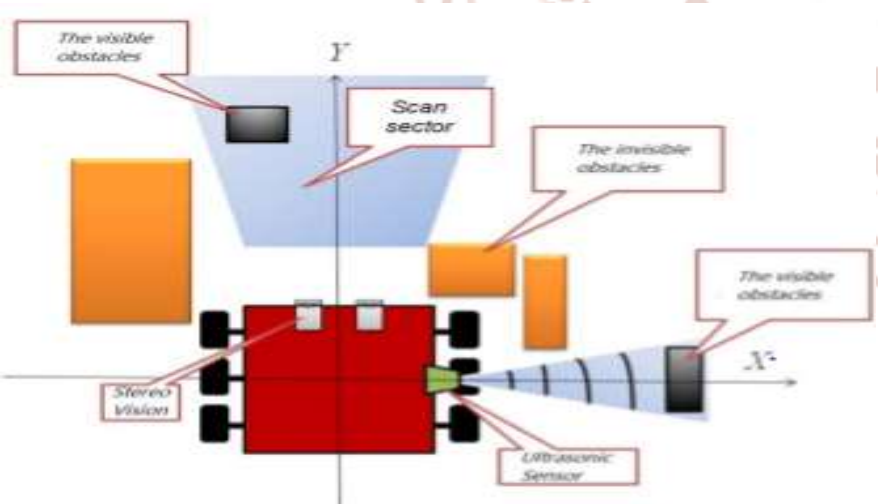

Figure 5: The robot movement within a territory guided by sensor

\section{C.SENSING MODULE:}

In this paper, the sensing module plays an important role in various ways. The first and foremost activity is to guide the robot in the rough terrain by detecting the obstacles. In fact, this turns out to be a self- learning concept in which the robot updates the map of a particular territory within its reach by means of the information obtained from the sensors [14]. The next important task is to sense the environmental conditions which include temperature, humidity gas, smoke etc. the readings obtained from these sensors will help the controller to analyze the situation in the Warfield and forward the soldiers towards the Warfield with precautionary measures[15].The next important activity is to find the enemy soldiers in the Warfield and the number of them who are present there hence the required tactics to manage with them and prior decisions can be made from this information. Hence the controller present at a certain distance can aggregate the information obtained from various sources and guide the soldiers accordingly.

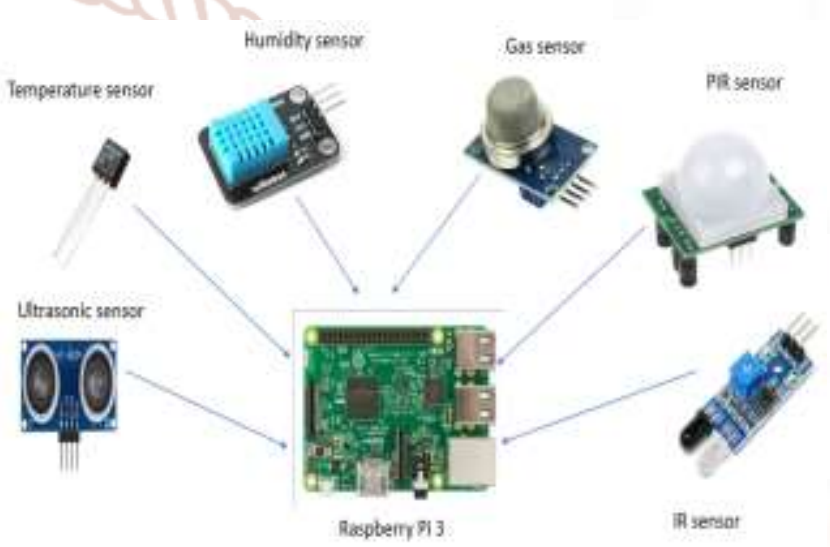

Figure 6: Sensors interfaced with Raspberry pi 3

V. HARDWARE REQUIREMENTS AND IMPLEMENTATION

The Raspberry pi 3 Model-B is the $3^{\text {rd }}$ generation Raspberry pi minicomputer with a $64-$ bit $1.2 \mathrm{GHz}$ quad-core processor, 1GB RAM, It also has $4 \mathrm{x}$ USB 2.0 ports, $10 / 100$ Ethernet, 40 GPIO pins, Full-size HDMI 1.3a port, Camera interface (CSI), Combined $3.5 \mathrm{~mm}$ analog audio and composite video jack, a Display interface (DSI), MicroSD slot and Video Core IV multimedia/3D graphics core@ 400MHz/300MHz.

$>$ Wi-Fi and Bluetooth 4.1 controllers

> The Raspberry pi Camera V1 is 5MP static sensitive type camera. It is a small PCB on which an Omni-vision OV5647 camera module. The pi camera module is connected to CSI port of Raspberry pi. A Passive Infra-Red (PIR) sensor is a pyro-electric device which detects level of IR radiation from the living objects.

A Passive Infra-Red (PIR) sensor is a pyroelectric device which detects level of IR radiation from the living objects. It has a specification of Sr501. 
$>$ Temperature Sensor, Humidity Sensor, IR sensor, Gas sensor, Ultrasonic sensor.

$>$ The motors assembly includes the Robot chassis and two DC geared motors. The L293D IC is a dual H-bridge motor controller, which is typically used to control the motor speed and direction[16]. This driver module is supplies a high current and high voltage to connected DC motors.

\section{SOFTWARE REQUIREMENTS IMPLEMENTATION}

AND

\section{A. Python Source Code for Raspberry Pi}

The entire system is based on a Raspbian operating system like Linux platform. The Raspbian OS is based on Debian that optimizes the Raspberry Pi hardware. The programming language which is utilized for coding is Python. Python referred to as a widely used high-level programming language for general-purpose programming. Python language is created by Guido van Rossum and was released in 1991. Besides Python is interpreted language which has a design philosophy that emphasizes code readability. It uses white space indentation to delimit code blocks rather than keywords or curly braces. A syntax which permits users to express concepts in lesser lines of code than possible in languages such as Java or $\mathrm{C}++$. Python programming language has a compiler that runs automatically when the Python source code (.by file) is executed in the terminal, and the .pic file is generated. The Figure 3 illustrates the software design flow of spy robot for a surveillance system. Basically, the functionality of system clarifies the cyclic phase rotation for robot movement. From the flowchart, the invoking sequence and the relationship between various functions are visualized.

\section{B.HTML JavaScript}

HTML provides the basic structure of sites, which is improved and customized by other technologies like CSS and JavaScript. CSS is used to control presentation, layout and formatting. JavaScript is used to control the performance of different elements. Bootstrap is the most popular HTML, CSS, and JavaScript front end framework for developing web pages, mobile web sites and more [17]. Bootstrap is completely free to use. The Internet of Things (IoT) can be considered as a global network which enables the communication between human to human, human to things and things to things. It is anything in the world by providing unique identity to each and every object. The commands can be sent through the web page with the help of internet. The user control command can be sent from anywhere in the world through web page. The robot is controlled from remote place which isolates the human being from dangerous environments.

As the main code is being executed, first, the robot will move forward, checks for human-being in the field, and IR sensors checking for obstacles might come before the robot, the robot will do these processes simultaneously. When the user giving inputs from the webpage, it is stored in server as a text file. At robot end the Raspberry pi running the python script which will read the text file and execute the command according the user inputs. The Raspberry pi is connected the H-bridge IC L293D which will control the direction of motors.

\section{BLOCK DIAGRAM \& WORKING}

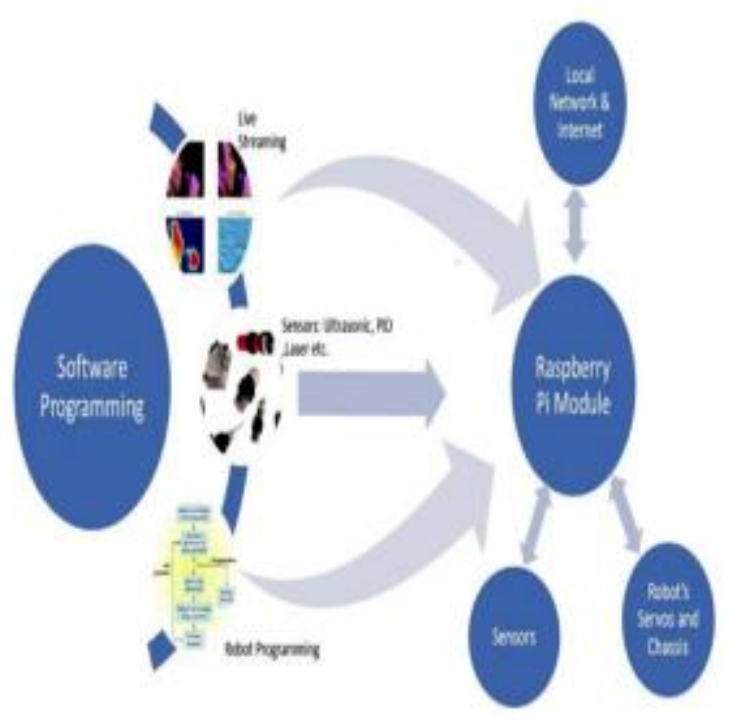

Figure 7: Block diagram for a Raspberry pibot

This is the proposed block diagram. This can tell in one glance about how the title can be proceeded. The raspberry pi being the center and the core of this, for making the robot and is its control unit. The pi is placed on the robot chassis which is connected by servos. The servos are connected to the raspberry pi via a switching circuit. This is a normal switching circuit which is used to make a robot. This switching circuit consists of the relay which is activated via our Web GUI whenever the user directs the robot to move it forward, reverse or for that matter any direction which will trigger the relay and the servos will start and work accordingly. Valeriu, Florin and AdrianViorel [18] research paper entitled "Control System for Video Advertising Based on Raspberry Pi" 
describes the implementation of server client model for controlling the robot made in the java platform.

In this Pibot i.e. the raspberry pi-controlled robot is having a server-client model. The server -client is made completely in java and will be able to run on any system be it windows, mac or Linux. This feature of being platform independent is the key reason to make this model in java. The Web GUI used to control the pi is also created using java and then linked to the server made. Widodo Budiharto [19]. research paper entitled "Design of Tracked Robot with Remote Control" in 2014, he introduced a surveillance system which is remotely controlled with the help of controller by a means that does not restrict its motion and able to acquire the live feeds of surrounding environment, for example in rescuing disaster victims.

This complete model of Pibot will be connected to the local network and can be controlled via anyone, anytime, \& anywhere. This Local network can be any place like home, office, prisons or for that matter anywhere which needs to have a temporary surveillance or a continuous one just plugs it in the network and the robot is good to go. This connection will be done via wireless network made, created or available at that place and made available to pi via Nano Wi-Fi adapter. The live streaming is being done by the help of MJPEG Streamer. It will be installed in the pi and then initializing the camera module. This camera module is the one designed by the raspberry pi organization for raspberry pi specially. It's a 1080p $30 \mathrm{fps} 5$ megapixel camera. The MJPEG Steamer uses the concept of time lapse photography to stream the video. It takes photos at a periodic interval and then overwrites one over other to make it look like a continuous stream of video. Due to the computational power of pi we have to choose this method for streaming video.

To keep the robot safe, it has been interfaced with an infrared sensor to it so that to avoid collision to any object if the person controlling it tends to or by mistake attempts to collide it somewhere. It has two servo motors of $30 \mathrm{rpm}$ each. The servo motors are interfaced to the raspberry pi via a switching circuit. GPIO Pin 6,11,12 are used for interfacing the motors, where pin 6 is ground while pin 11,12 are general input/output pins. The switching circuit basically comprise of a microcontroller IC AT89c51 and three relay switch each for individual motor and third one for the infrared sensor to stop it if an obstacle comes in its way. It has three $9 \mathrm{~V}$ batteries being used for the motors and the infrared sensor each. The raspberry pi is being

Powered by a 10400mAh power bank having a constant output of 5V 1A. Brian et al., 2014 research paper entitled "Sudo Pi Cooler / Heater" describes a typical temperature sensing device in which temperature of an area is recorded and is adjusted based upon the preset values [20]. The temperature sensor using raspberry pi aims to provide an adaptable temperature sensing approach using a Raspberry Pi. So, the user can interface various other sensors too to get more data of the environment in which it is used like the temperature sensor, $\mathrm{CO} 2$ sensor for mentoring the carbon di-oxide content in that particular place.

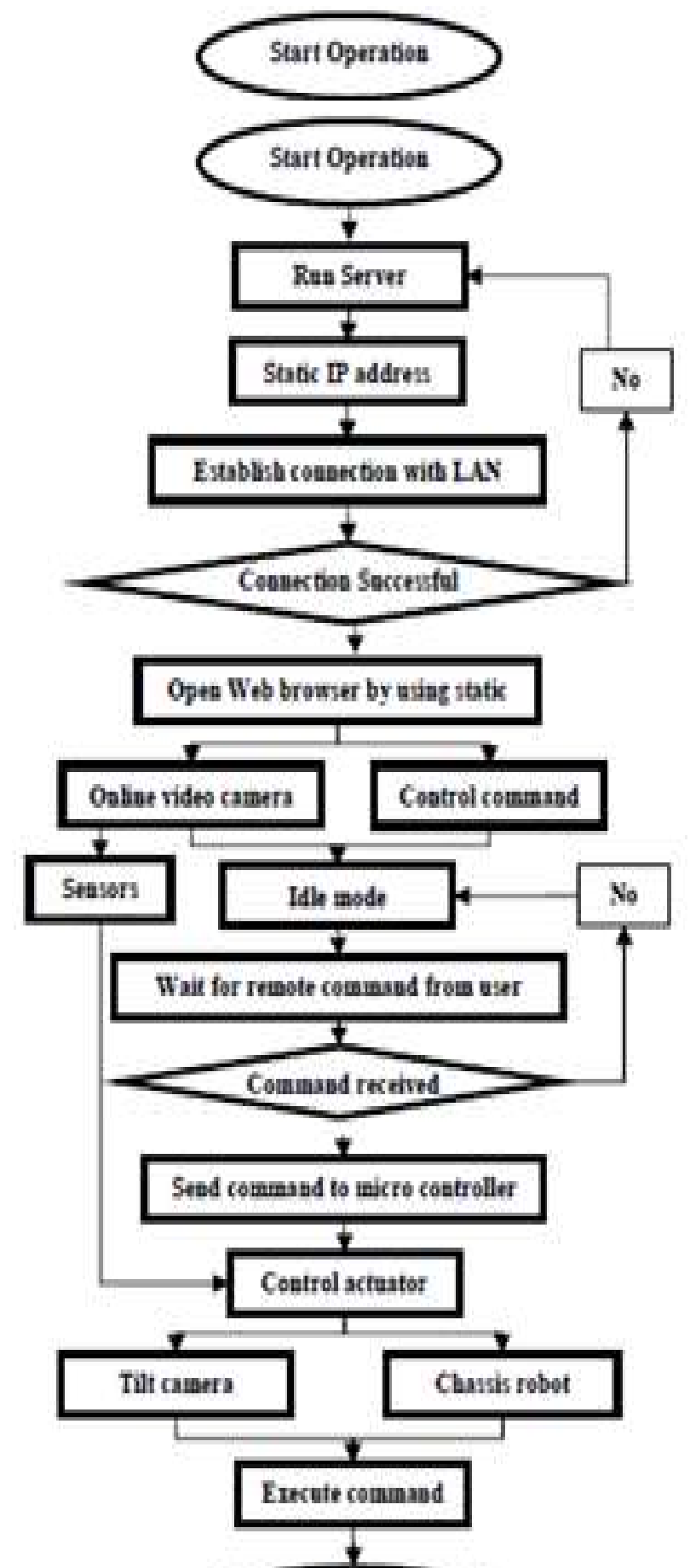


$>$ For configuring the robot user has to go to system every time. This should be little hectic for user. Also considering that fact that this robot is not compatible with Windows operating system and cannot install it which most of the users are familiar to use.

\section{PROS AND CONS}

It is important for users that the Pibot fits with their utilities and are willing to get this robot and tailoring the product to their own needs. Generally, every

project does have some advantages or disadvantages. Surely this project also has some pros and cons:

\section{PROS:}

$>$ It is fully dependent on the Raspberry Pi which is a micro-computer [21]. This robot is useful for the organization where they can't afford the costly surveillance systems.

$>$ Compact size and portable - used in any kind of surrounding for surveillance.

$>$ Skid-steered mechanism maintains the friction and establish equilibrium while in move.

$>$ If the user think that it is based on Raspberry Pi technology and user should have the extensive command on programming, then NO. The robot comes with the all utilities and software's required for live streaming and surveillance so that the user can use it without any hesitation or fear that they might not be able to control it.

$>$ This surveillance robotic system will provide a lot enhanced feature over analog and the cost of the device will also be efficient during mass production [22][23].

\section{CONS:}

As raspberry Pi does not have an integrated Wi-Fi. User has to buy the Wi-Fi adapter or something like that to connect to the network wirelessly.

$>$ This robot can occupy only one location for surveillance at a time.

$>$ This robot can do work in only local network, user can't connect robot via internet.

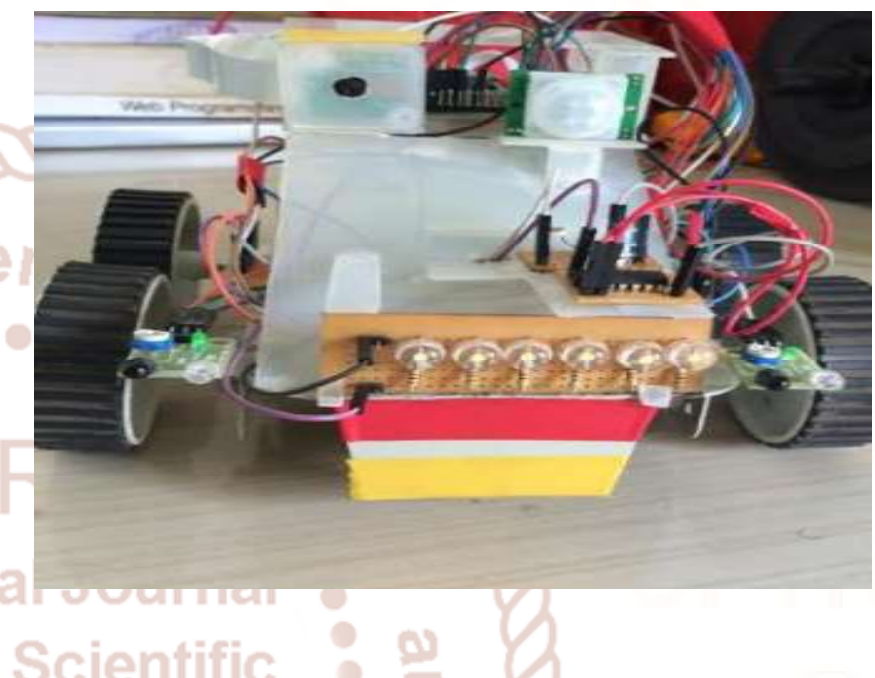

Figure 9. The front view of the Pi Robot for a surveillance system

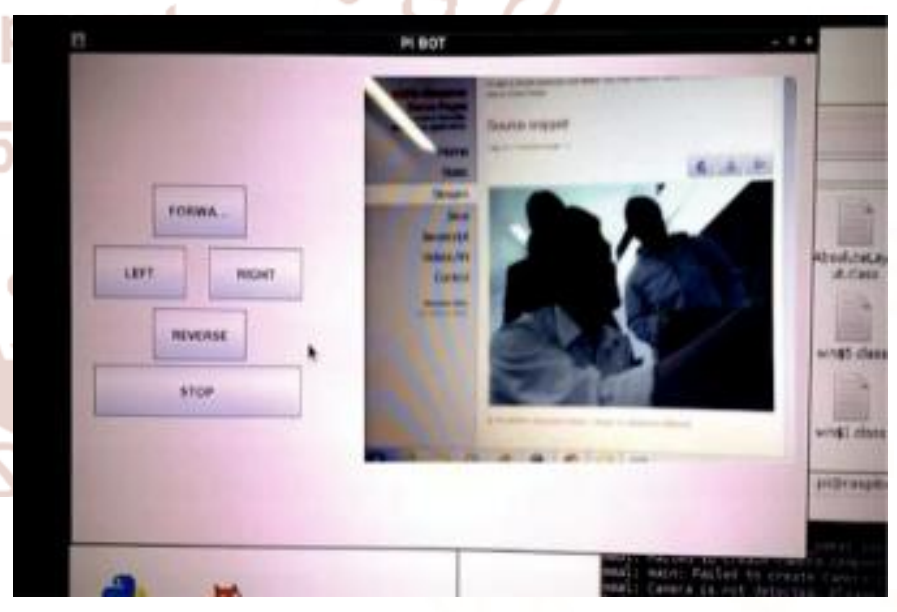

Figure 10: Live streaming \& Web-GUI for controlling the robot

\section{IX .CONCLUSION}

In this paper we have illustrated the capabilities of raspberry pi over arduino and advantages of the pibot over the conventional surveillance systems. The System proposed is a low-cost and efficient smart Surveillance Pi based robotic System which will be able to record / capture video/image and transmit the 
necessary information through the internet. Pi robot can be easily controlled using the personal computer or a smart phone. It is beneficiary as it offers reliability and privacy on both sides. The web page created to control the robotic movements is secured by unique user name and password which cannot be hacked easily. This will enhance the privacy and security in the proposed model.

\section{FUTURE SCOPE}

This project can be extended further by making the robot accessible via the internet. This can be implemented by making a android/iOS/windows phone app and then controlling it via the same. The robot can also made to implement the SLAM (simultaneous localization and mapping algorithms) to make it map the complete environment and then move autonomously after a certain periodic intervals to check everything. Also by giving it the ability to detect and recognize faces it can be made to alert us about any unknown person and take a snap of it and email us the same. It can be made to follow a specific face continuously rather than manually operate it to follow someone like the small children in the age group of1-4 years so that kids are continuously in front of our eyes. Can also take help of sensors to maintain a safe distance from the kids for the safety of the robot

\section{REFERENCES}

1) A.S.M.MdSaifur Rahman, Md Fahim-Al-Fattah, Md Arifur Rahman ZadidShifat, "A Practical Approach to Microcontroller Based Smart Phone Operated Robotic System at Emergency Rescue Scheme," 9th International Forum on Strategic Technology (IFOST), pp. 414-417, 2014.

2) Keerthi Premkumar, and K Gerard Joe Nigel, "Smart Phone Based Robotic Arm Control Using Raspberry Pi, Android and Wi-Fi," IEEE Sponsored 2nd International Conference on Innovation in Information Embedded and Communication Systems, 2016

3) Pavithra.S and S.A. SivaSankari, "7th Sense-A Multipurpose Robot for Military," International Conference In Information Communication and Embedded Systems (ICICES), pp. 1224-1228, 2013.

4) Charles Severance, "Eben Upton: Raspberry Pi”, Published by IEEE computer society, October 2013.
5) Kawser Jahan Raihan, Mohammad Saifur Rahaman,Mohammad Kaium Sarkar \&SekhMahfuz , "Raspberry Pi Image Processing Based Economical Automated TollSystem,2013.

6) Gareth Mitchell, The Raspberry Pi single-board computer will revolutionize computer science teaching [For \& against], Vol.7, NO.3, pp. 26, 2012 .

7) Matt Richardson and Shawn Wallace, Getting Started with Raspberry Pi. United States of America: O’Reilly Media, 2013.

8) M. Vanitha, M. Selvalakshmi, R. Selvarasu Monitoring and controlling of mobile robot via internet through raspberry Pi board. IEEE Xplore: 08 September 2016

9) S. Kumar, S. Raghavendra, M. Bhagat, K.V. Gangadharan, "Design, simulation and testing of shrimp rover using recurdyn", 12th Symposium on Advanced Space Technologies in Automation and Robotics, May 2013.

10) K. Iagnemma, A. Rzepniewski, S. Dubowsky, "Control of robotic vehicles with actively articulated suspensions in rough terrain", Autonomous Robots, vol. 14, issue 1, pp. 516, Jan. 2003.

11) V. Mazulina, G. Mishchenko, "The identification and avoidance of the obstacles on the mobile robot way using the technical vision", The Almanac of the young scientists' scientific, ITMO University, vol. 2, pp. $164-167,2015$.

12) M. Evstigneev, V. Mazulina, G. Mishchenko, Y. Litvinov, "Algorithms of control over for-wheel robot moving over rough terrain", Journal of instrument engineering, vol. 58, issue 9, pp. $738-$ 741, Sept. 2015.

13) V. Mazulina, Y. Litvinov, S. Frolov, I. Salmygin, A. Bushuev, "Using of the web - camera for the obstacles detection to the movement of the mobile robot", Information processing systems. Information problems of acoustic, electronic and telecommunication systems theory, issue 7, pp. 24 $-26,2013$

14) A. U. Bokade and V. R. Ratnaparkhe, "Video surveillance robot control using smartphone and Raspberry pi," 2016 International Conference on Communication and Signal Processing (ICCSP), Melmaruvathur, 2016, pp. 2094-2097. 
15) W. F. Abaya, J. Basa, M. Sy, A. C. Abad and E. P. Dadios, "Low cost smart security camera with night vision capability using Raspberry Pi and OpenCV," 2014 International Conference on Humanoid, Nanotechnology, Information Technology, Communication and Control, Environment and Management (HNICEM), Palawan, 2014, pp. 1-6.

16) S.H. Chia, H. Guo, B.Y. Li, et al, Team mobile robots based intelligent security system, Applied mathematics \& information science, 7(2013) 435440

17) S. Agamah and L. Ekonomou, "A PHP Application Library for WebBased Power Systems Analysis," 2015 IEEE European Modelling Symposium (EMS), Madrid, 2015, pp. 353-358.

18) G. Qiao, G. Song, Y. Wnag et al, Autonomous network repairing of a home security system using modular self-reconfigurable robots, IEEE Trans. on Consumer Electronics, 59(2013) 562-570
19) Widodo Budiharto, "Design of Tracked Robot with Remote Control", 2014.

20) Valeriu, Florin and Adrian-Viorel, "Control System for Video Advertising Based on Raspberry Pi”, 2014.

21) S.H. Chia, H. Guo, B.Y. Li, et al, Team mobile robots based intelligent security system, Applied mathematics \& information science, 7(2013) 435440.

22) S.A. Li,Y.CH. Lin, CH.V. Weng, et al, Circle object recognition based on monocular vision for home security robot, Conf.IEEE ISPACS, New Taipei, 2012, 732-738.

23) J.Y. Jung, B.K. Dan, K.H. An, et al, Real-time human tracking using fusion sensor for home security robot, Conf. IEEE ICCE, Las Vegas, $2012,420-425$ 